\title{
In-plane and out-of-plane thermal conductivity of a large single crystal of $\mathrm{YBa}_{2} \mathrm{Cu}_{3} \mathrm{O}_{7-x}$
}

\author{
M. Matsukawa, T. Mizukoshi, and K. Noto \\ Faculty of Engineering, Iwate University, 4-3-5 Ueda, Morioka 020, Japan \\ Y. Shiohara \\ Superconductivity Research Laboratory, ISTEC, 1-10-13, Shinonome, Koto-ku, Tokyo 135, Japan
}

(Received 11 December 1995)

\begin{abstract}
The in-plane and out-of-plane thermal conductivity, $\kappa_{a b}$ and $\kappa_{c}$, of a large single crystal of twinned $\mathrm{YBa}_{2} \mathrm{Cu}_{3} \mathrm{O}_{7-x}$ has been studied as a function of temperature for several annealing conditions. The present result strongly supports ascribing an observed peak in $\kappa_{a b}$ below $T_{c}$ to an electron contribution. The superconducting enhancement of $\kappa_{a b}$ becomes rather small and the position of the peak value gradually shifts to higher temperatures with an increase of annealing time. This behavior is not well explained by the KadanoffMartin expression on the assumption of Matthiessen's rule but probably implies that the screening effect due to charge carrier doping yields a suppression of strong electron-electron interaction in the normal state.
\end{abstract}

Recently, a number of studies on the thermal conductivity $\kappa$ of high- $T_{c}$ oxide materials have been reported. ${ }^{1-4}$ A common feature in their studies is that high- $T_{c}$ oxide superconductors, except for the 2-1-4 system such as $\mathrm{La}_{2-x} \mathrm{Sr}_{x} \mathrm{CuO}_{4}$ and $\mathrm{Nd}_{2-x} \mathrm{Ce}_{x} \mathrm{CuO}_{4}$ crystals show an anomaly in the thermal conductivity associated with the superconducting transition. An origin of such an anomaly in $\kappa$ is not made clear yet but two scenarios have been presented to interpret the observed anomaly. The measured thermal conductivity is separated into phonon and electron components. One scenario attributes the origin of the anomaly to a phonon contribution, while in an alternative scenario the observed anomaly is ascribed to an electron contribution. ${ }^{5-8}$

In this paper, the in-plane and out-of-plane thermal conductivity, $\kappa_{a b}$ and $\kappa_{c}$, of a large single crystal of twinned $\mathrm{YBa}_{2} \mathrm{Cu}_{3} \mathrm{O}_{7-x}$ (YBCO) has been studied as a function of temperature for several annealing conditions. A measured single crystal was prepared by a modified Czochralski method called the solute rich liquid crystal pulling method (the SRL-CP method). The details of the SRL-CP method are reported in Ref. 9. Sample dimensions are typically $4.5 \times 3.1$ $\mathrm{mm}^{2}$ in the $a b$ plane and $1.7 \mathrm{~mm}$ along the $c$ axis. The largest single crystal was used for the thermal conductivity measurement as far as we know. The x-ray Laue patterns indicated a very high-quality crystal. The as-grown sample was annealed in oxygen flow at $520^{\circ} \mathrm{C}$ for three days, one week, and two weeks (sample Nos. 1, 2, and 3). For each annealing condition, the thermal and electrical conductivity measurements were performed on the same crystal. The thermal conductivity measurement was made from 10 up to $150 \mathrm{~K}$ with a steady-state heat-flow method using a fully automated measurement system with a helium refrigerator. ${ }^{10}$ The heat flow is in the $a b$ plane or along the $c$ axis, and a temperature gradient of $\sim 1 \mathrm{~K} / \mathrm{cm}$ is monitored in a steady state by a differential $\mathrm{Au}+0.07$ at. \% Fe alloy-Chromel thermocouple. For the $\kappa_{c}$ measurement, the sample was sandwiched between two sapphire substrates, and the thermocouple junctions were attached to these substrates with a GE 7051 varnish. The detailed configuration is described in Ref. 2. The electrical conductivity was measured with a dc four-probe method.
The in-plane thermal conductivity $\kappa_{a b}$ of a large single crystal of twinned YBCO is shown in Fig. 1 as a function of temperature from 10 to $150 \mathrm{~K}$ for several annealing conditions. The $\kappa_{a b}$ value of an annealed sample shows a clear enhancement just below $T_{c}$ with decreasing temperature and forms a rapid peak at $\sim 40 \mathrm{~K}$, while the $\kappa_{a b}$ value of an as-grown sample exhibits no pronounced anomaly below $T_{c}$. The normal-state $\kappa_{a b}$ value for all samples shows almost the same temperature dependence and weakly decreases with increasing temperature, which is probably associated with phonon-phonon Umklapp scattering. The observed peak value $\kappa_{a b}{ }^{\max }$ is enhanced and its position gradually shifts to higher temperatures with increasing annealing time. Furthermore, the value of $\kappa_{a b}{ }^{\max } / \kappa_{a b}(100 \mathrm{~K})$ becomes rather small and the anomaly in $\kappa_{a b}$ associated with the superconducting transition is weakly depressed for long-time annealing. ${ }^{11} \mathrm{Of}$ course, for short-time annealing, the value of $\kappa_{a b}{ }^{\max } / \kappa_{a b}$ $(100 \mathrm{~K})$ for a single-crystal YBCO is enchanced and the peak value position shifts to lower temperatures as reported in Ref. 11. Figure 2 shows the out-of-plane thermal conductivity $\kappa_{c}$ of a large single crystal of YBCO as a function of

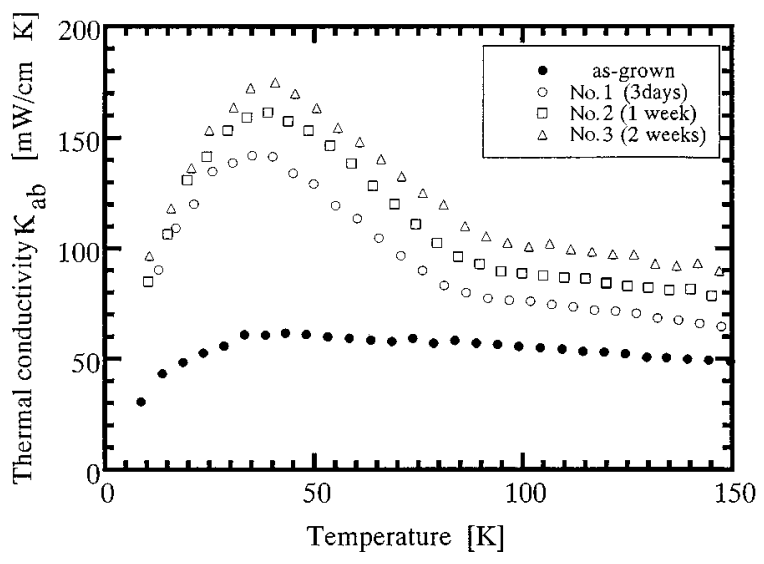

FIG. 1. In-plane thermal conductivity $\kappa_{a b}$ of a large single crystal of twinned YBCO plotted as a function of temperature for several annealing conditions. 


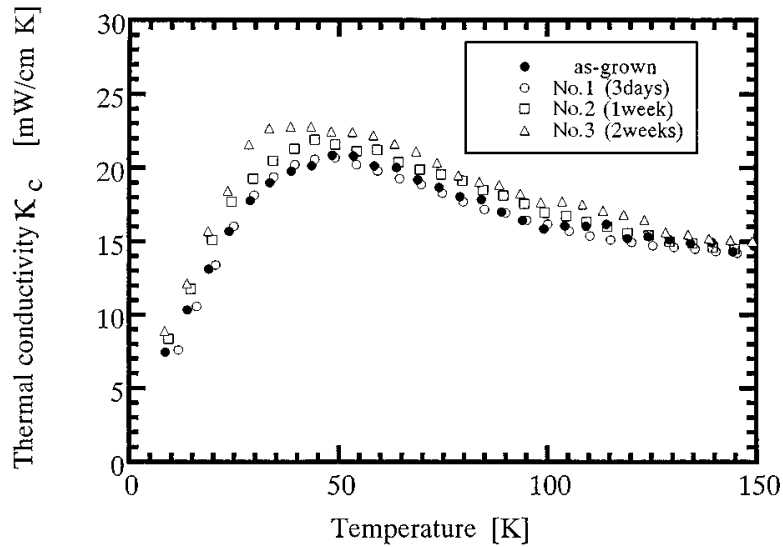

FIG. 2. Out-of-plane thermal conductivity $\kappa_{c}$ of a large single crystal of twinned YBCO plotted as a function of temperature for several annealing conditions.

temperature from 10 to $150 \mathrm{~K}$ for several annealing conditions. The $\kappa_{c}$ value for all samples increases gradually from $150 \mathrm{~K}$ with decreasing temperature and shows no remarkable anomaly below $T_{c}$. The maximum value of $\kappa_{c}$ is weakly enhanced and its position shifts to lower temperatures with increase of annealing time. Such behavior is due to the decrease of phonon scattering by point-defects because the annealing process decreases the oxygen deficiency. The measured value of $\kappa_{c}$ is comparable with the result of a small single crystal of YBCO obtained by Hagen et al. ${ }^{2}$ A negative curvature in $\kappa_{c}(T)$ originates in phonon-phonon Umklapp scattering, like that in $\kappa_{a b}$ above $T_{c}$. The in-plane and outof-plane resistivity of $\rho_{a b}$ and $\rho_{c}$ of a large single crystal of YBCO is shown in Fig. 3 as a function of temperature from 10 to $300 \mathrm{~K}$ for several annealing conditions. The temperature dependence of $\rho_{a b}$ for the annealed samples shows a metallic behavior down to the onset of $T_{c}$ and the value of $T_{c \text {,zero }}$ is determined to be $92.7 \mathrm{~K}$ from the resistivity data except for the as-grown sample. The value of $T_{c \text {,zero }}$ remains constant, but the residual resistivity at $0 \mathrm{~K}, \rho_{a b}(0)$ obtained from the $T$-linear extrapolation of $\rho_{a b}$ reaches a minus value for a two week annealed sample. On the other hand, the

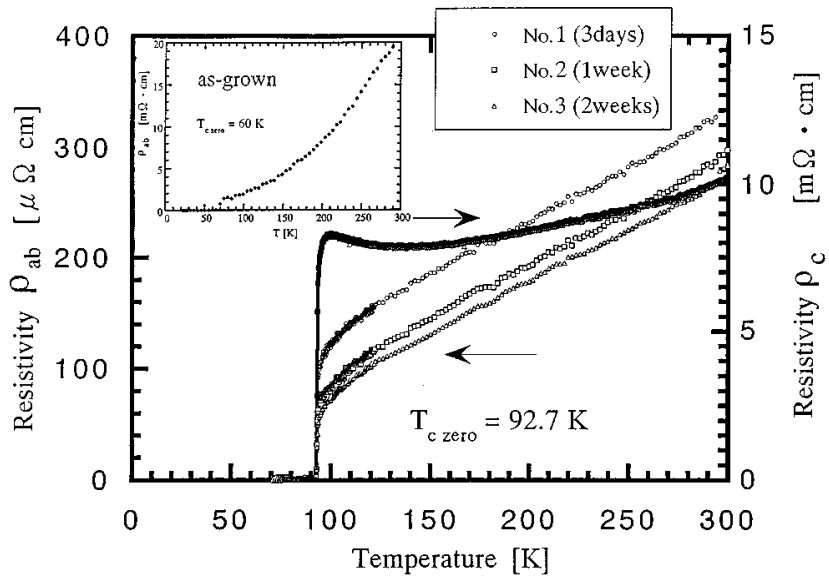

FIG. 3. In-plane and out-of-plane resistivity, $\rho_{a b}$ and $\rho_{c}$, of a large single crystal of $\mathrm{YBCO}$ as a function of temperature from 10 to $300 \mathrm{~K}$ for several annealing conditions. The inset shows the temperature dependence of $\rho_{a b}$ for the as-grown sample. temperature dependence of $\rho_{c}$ exhibits a semiconducting behavior and the value of $\rho_{c}(T)$ and $T_{c \text {,zero }}$ are independent of the annealing time.

The measured thermal conductivity is separated into phonon and electron contributions $\kappa_{\mathrm{ph}}$ and $\kappa_{\mathrm{e}}$. The electronic thermal conductivity in the normal state $\kappa^{n}{ }_{\mathrm{e}}$ is estimated from the electrical resistivity data using the WiedemannFranz law (WF law). ${ }^{12}$ The estimated value of $\kappa^{n}{ }_{a b, \mathrm{e}} / \kappa_{a b}$ for annealed samples ranges from 31.5 to $32.7 \%$ at $150 \mathrm{~K}$, but the ratio of $\kappa^{n}{ }_{c, \mathrm{e}} / \kappa_{\mathrm{c}}$ for all samples becomes a very small value of $\sim 3.1 \%$ at $150 \mathrm{~K}$ and is negligible. The thermal transport carriers in the $a b$ plane are both phonons and electrons except for the as-grown sample while thermal carriers along the $c$ axis are only phonons. For the as-grown sample, the value of $\kappa^{n}{ }_{a b, \mathrm{e}}$ is also negligible and the measured value of $\kappa_{a b}$ corresponds to the phonon thermal conductivity. Subtracting the $\kappa_{a b}$ value of the as-grown sample from that of the annealed one for three days, the obtained difference $\Delta \kappa_{a b}\left[=\kappa_{a b}\right.$ (sample No. 1) $-\kappa_{a b}$ (as grown) $]$ is in good agreement with the electronic thermal conductivity $\kappa_{a b, \mathrm{e}}^{n}$ of sample No. 1 estimated from the resistivity data of $\rho_{a b}$ by the WF law. A similar discussion on the $a b$-plane thermal conductivity of insulating $\mathrm{Bi}_{2} \mathrm{Sr}_{2} \mathrm{YCu}_{2} \mathrm{O}_{y}$ and superconducting $\mathrm{Bi}_{2} \mathrm{Sr}_{2} \mathrm{CaCu}_{2} \mathrm{O}_{y}$ has been made by Allen et al. ${ }^{13}$ The value of $\kappa_{a b}\left(\approx \kappa_{a b, \mathrm{ph}}\right)$ for the as-grown sample is nearly equal to the phonon component of $\kappa_{a b}$ for sample No. 1 . This result indicates that carrier doping does not considerably change the phonon thermal conductivity and phonon scattering by charge carriers is very small compared with other scattering processes such as point-defect scattering and phonon-phonon Umklapp scattering. If electron-phonon interaction were dominant in the thermal transport of this material, phonon scattering by charge carriers would be introduced by carrier doping so that the phonon component would be strongly suppressed in the annealed sample. Therefore, it is difficult to interpret our experimental result on the basis of the electron-phonon interaction model proposed by Bardeen, Rickayzen, and Tewordt. ${ }^{5,6}$

Next, the electronic thermal conductivity in the superconducting and normal states for both annealed samples Nos. 1 and 3 is estimated from the $\kappa_{a b}$ data of the as-grown sample. The $\kappa_{a b, \mathrm{ph}}$ value of annealed samples No. 3 is obtained from the $\kappa_{a b}$ data of the as-grown sample by a small correction. The $\kappa_{c}$ value of sample No. 3 is larger than that of sample No. 1 by a factor of about 1.12 because phonon scattering by point defect is decreased by oxygen annealing. This variation is probably reflected not only in the $\kappa_{c}$ value but also in a phonon component of $\kappa_{a b}$. Thus, the $\kappa_{a b \text {,ph }}$ value of annealed samples No. 3 is scaled by a factor of about 1.12 to the $\kappa_{a b, \mathrm{ph}}$ value of annealed samples No. $1\left(\approx \kappa_{a b}\right.$ of the as-grown sample). As a result of a small modification, the temperature dependence of $\kappa_{a b, \mathrm{e}}$ for annealed samples Nos. 1 and 3 is shown in Fig. 4. In comparison, the $\kappa_{a b, \mathrm{e}}^{n}$ value in the normal state estimated from the WR law is also plotted.

We discuss the superconducting-state electronic thermal conductivity $\kappa_{a b, \mathrm{e}}$ of a single-crystal YBCO on the basis of a phenomenological model proposed by Yu et $^{\mathrm{al}} .^{7}$ The value of $\kappa_{e}^{s}$ is calculated in terms of the Kadanoff-Martin (KM) expression, 


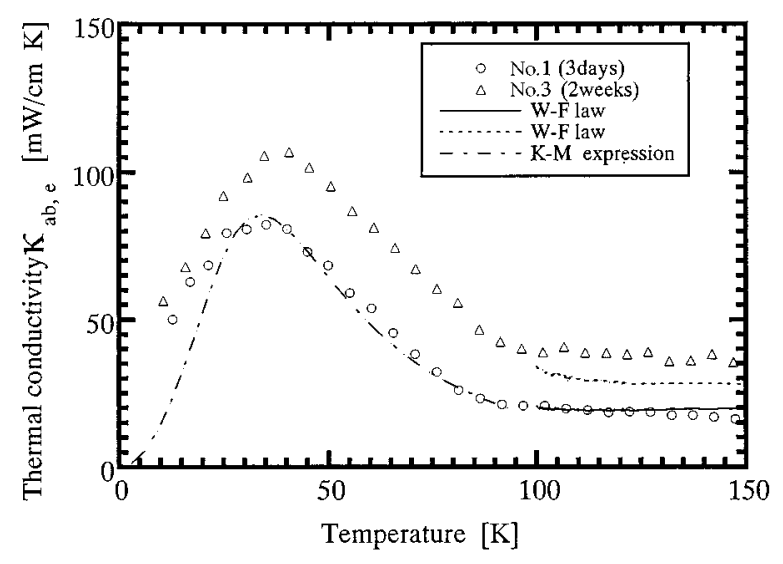

FIG. 4. Temperature dependence of $\kappa_{a b, \mathrm{e}}$ for the annealed samples Nos. 1 and 3. For comparison, the $\kappa_{a b, \mathrm{e}}^{n}$ value in the normal state estimated by the WF law is also plotted. A dot-dashed line shows a best fitted curve using the Kadanoff-Martin expression.

$$
\bar{\kappa}_{e}^{s}=\int_{0}^{2 \pi} \frac{d \phi}{2 \pi} \frac{3}{2 \pi^{2}} t \int_{0}^{\infty} d \varepsilon^{2} \operatorname{sech}^{2}(E / 2) \frac{1+a}{\epsilon / E+a t^{n}}
$$

with

$$
E=\sqrt{\varepsilon^{2}+\Delta^{2}}, \quad t=\frac{T}{T_{c}}
$$

and

$$
\Delta(t, \phi)=\chi \Delta_{\mathrm{BCS}}(t) \psi_{d}(\phi)
$$

where $\bar{\kappa}_{e}^{s}$ are normalized by the value of the electronic thermal conductivity at $T_{c} .{ }^{14}$ The variables of $\varepsilon$ and $\Delta(T)$ normalized by $k T$ denote the quasiparticle energy measured from Fermi-level and the superconducting energy gap according to the Bardeen, Cooper, and Schrieffer (BCS) theory, respectively. In the integrand of Eq. (1), the parameter of $a$ represents the ratio of a power-law scattering rate to the residual scattering rate, which roughly corresponds to the value of the residual resistivity ratio (RRR). In the calculation of the $s$-wave and $d$-wave pairing-states, the wave functions are taken as $\psi_{s}=1$ and $\psi_{d}=\sqrt{2} \cos (2 \phi)$, respectively, where $\phi$ is the azimuthal angle of wave number $k$ in the $a b$ plane. ${ }^{15}$ The value of $\chi$ is a scaling parameter, the ratio of the superconducting gap to the value of the BCS gap, i.e., $\chi=\Delta(0)$ / $\Delta_{\mathrm{BCS}}(0)$. In the normal state, the power-law value in the quasiparticle scattering rate is taken as $n=1$ because in a high$T_{c}$ superconductor such as the YBCO system, the $T$-linear dependence of the electrical resistivity gives the quasiparticle scattering rate proportional to $T$ on the assumption of the WF law. Here, the origin of the $T$-linear dependence of the quasiparticle scattering rate in the normal state is considered to be strong electron-electron interaction in the $\mathrm{CuO}_{2}$ plane. On the other hand, the quasiparticle scattering rate is strongly suppressed in the superconducting state since the strong electron-electron interaction in the normal state is rapidly reduced due to the formation of the superconducting gap in the excitation spectrum in the electron system. If the effect of a rapid suppression in the quasiparticle scattering rate becomes very predominant over the decrease of the quasiparti- cle number, then the temperature dependence of $\kappa_{e}$ shows a peak below $T_{c}$ as pointed out by Yu et al. ${ }^{7}$

A best fitted curve to $\kappa_{a b, \mathrm{e}}^{s}$ of sample No. 1 is obtained in the $d$-wave pairing state as shown in Fig. 4, where $n=4$, $a=50$, and $\chi=0.7$. The temperature dependence of $\kappa_{e}$ at low temperatures is well-described not by the $s$-wave pairing state with an isotropic superconducting gap but by the $d$-wave pairing state with an anisotropic one. However, within this calculation, it remains possible that the $s$-wave pairing state with anisotropic gap structure exists. The power-law value is the same one as obtained for a single crystal of untwinned YBCO. The fitted value of $a$ is five times as large as the RRR value of 9, estimated from the $T$-linear extrapolation of the resistivity data to $0 \mathrm{~K}$. The resistivity of annealed sample No. 3 shows the super $T$-linear dependence and then the residual resistivity $\rho_{a b}(0)$ takes a negative value. However, it should be noted that the value of RRR ( $\approx$ the parameter of $a$ ) never takes a negative value for the conventional metals and alloys. Thus, on the assumption of Matthiessen's rule in the KM expression it is not possible to analyze the temperature dependence of $\kappa_{a b, \mathrm{e}}^{s}$ of sample No. 3. The position of peak value $\kappa^{\max }{ }_{a b, \mathrm{e}}$ shifts to higher temperature and the value of $\kappa^{\max }{ }_{a b, \mathrm{e}} / \kappa_{\mathrm{ab}, \mathrm{e}}(100 \mathrm{~K})$ decreases from 4.1 (sample No. 1) to 2.9 (sample No. 3) when annealing time increases. Heat treatment for a long time yields a decrease of quasiparticle scattering by the impurity so that it is expected that the position of $\kappa^{\max }{ }_{a b, \mathrm{e}}$ would shift to lower temperature and the value of $\kappa_{a b, \mathrm{e}}^{\max _{a, \mathrm{e}} / \kappa_{a b, \mathrm{e}}}(100 \mathrm{~K})$ increases. However, the present experimental result conflicts with such an expectation. This fact means that Matthiessen's rule breaks down not only in electrical conductivity but also thermal conductivity for high- $T_{c}$ materials. Of course, it should be noted that this change due to oxygen annealing does not originate in a variation on the phonon component of $\kappa_{a b}$ because the peak value of $\kappa_{c}$ shifts to low temperatures with increasing annealing time. The cause of this variation is explained as follows. An observed enhancement in $\kappa_{a b}$ below $T_{c}$ is caused by the freezing out of strong electronelectron interaction in the normal state due to formation of the superconducting energy gap within the scattering spectrum of electron system. In this case, the strength of electronelectron interaction is reflected to such enhancement, in other words, the value of $\kappa^{\max }{ }_{a b, \mathrm{e}} / \kappa_{a b, \mathrm{e}}(100 \mathrm{~K})$. A smaller value of $\kappa^{\max }{ }_{a b, \mathrm{e}} / \kappa_{a b, \mathrm{e}}(100 \mathrm{~K})$ means qualitatively a suppression of electron-electron interaction if the strength of electron scattering by impurities is invariant. Thus, it is supposed that strong electron-electron interaction becomes suppressed because of the screening effect due to carrier doping in oxygen anneal. In a previous paper, it has been reported that an annealing time of more than five days at $500{ }^{\circ} \mathrm{C}$ is needed to obtain an almost homogeneous oxygen distribution within a single crystal of YBCO with a size of $1 \mathrm{~mm}$ grown by the SRL-CP method. ${ }^{16}$ Taking account of the size of our sample and the fact that the oxygen diffusion along the $c$ axis of the sample is negligible with that in the $a b$ plane, an annealing time of more than 20 days is needed for obtaining a homogeneous carrier concentration. Furthermore, the $T_{c}$ value observed in resistivity is determined from a small volume fraction of superconductivity and gives no information about oxygen inhomogeneity in a large single crystal. Accordingly, it is believed that a change of carrier concentration possibly 
occurs and carrier concentration is not saturated for an annealing time up to two weeks, although the $T_{c, \text { zero }}$ value is not changed.

In summary, the in-plane and out-of-plane thermal conductivity, $\kappa_{a b}$ and $\kappa_{c}$, of a large single crystal of twinned YBCO has been studied as a function of temperature for several annealing conditions. The present result strongly supports a picture in which the observed peak in $\kappa_{a b}$ below $T_{c}$ is ascribed to the electron contribution. The superconducting enhancement of $\kappa_{a b}$ becomes rather small and the position of the peak value gradually shifts to higher temperatures with an increase of the annealing time. This behavior is not well explained by the KM expression on the assumption of Matthiessen's rule but implies that the screening effect due to charge carrier doping yields a suppression of strong electronelectron interaction in the normal state.

We are grateful to Professor N. Kobayashi at Institute for Materials Research, Tohoku University and Professor K. Yoshida at the Institute of Atomic Energy, Kyoto University for their valuable discussions. This work was partially supported by NEDO for the research and develoment of Industrial Science and Technology Frontier Program.
${ }^{1}$ See for a review paper, C. Uher, J. Supercond. 3, 337 (1990).

${ }^{2}$ S. Hagen, Z. Z. Wang, and N. P. Ong, Phys. Rev. B 40, 9389 (1989).

${ }^{3}$ M. F. Crommie and A. Zettle, Phys. Rev. B 41, 10978 (1990).

${ }^{4}$ A. V. Inyushkin, A. N. Taldenkov, L. N. Dem'yanets, T. G. Uvarova, and A. B. Bykov, Physica B 194-196, 479 (1994).

${ }^{5}$ J. Bardeen, G. Rickayzen, and L. Tewordt, Phys. Rev. 113, 1014 (1959).

${ }^{6}$ L. Tewordt and T. H. Wölkhausen, Solid State Commun. 70, 839 (1989).

${ }^{7}$ R. C. Yu, M. B. Salamon, Jian Ping Lu, and W. C. Lee, Phys. Rev. Lett. 69, 1431 (1992).

${ }^{8}$ P. B. Littlewood and C. M. Varma, J. Appl. Phys. 69, 4980 (1991).
${ }^{9}$ Y. Yamada and Y. Shiohara, Physica C 217, 182 (1993).

${ }^{10}$ M. Matsukawa, F. Tatezaki, K. Noto, H. Fujishiro, K. Michishita, and Y. Kubo, Cryogenics 34, 685 (1994).

${ }^{11}$ J. L. Cohn, E. F. Skelton, S. A. Wolf, J. Z. Liu, and R. N. Shelton, Phys. Rev. B 45, 13144 (1992).

${ }^{12}$ L. B. Ioffe and G. Kotliar, Phys. Rev. B 42, 10348 (1990).

${ }^{13}$ P. B. Allen, X. Du, and L. Mihaly, Phys. Rev. B 49, 9073 (1994).

${ }^{14}$ L. P. Kadanoff and P. C. Martin, Phys. Rev. 128, 2390 (1961).

${ }^{15}$ C. T. Rieck, D. Fay, and L. Tewordt, Phys. Rev. B 41, 7289 (1990).

${ }^{16}$ S. Miyamoto, Y. High, J. Schutzmann, H. Kutani, Y. Yamada, S. Tajima, and Y. Shiohara, in Advances in Superconductivity VII, edited by K. Yamafuji and T. Morishita (Springer, Berlin, 1994), Vol. 1, p. 129. 